\title{
Mass Mimicking Autoimmune Pancreatitis-A Report of Two Cases and Review of Literature
}

\author{
Diva S. Shah ${ }^{1}$ Bharat Prajapati ${ }^{2}$ Kintan Sanghavi ${ }^{3}$ \\ Jignesh Dubal ${ }^{4}$ \\ ${ }^{1}$ Department of Radiology, HCG Cancer Centre, Ahmedabad, \\ Gujarat, India \\ ${ }^{2}$ GI Onco-Surgery Department, HCG Cancer Centre, Ahmedabad, \\ Gujarat, India \\ ${ }^{3}$ Department of Histopathology and Cytology, Strand Life Science, \\ Ahmedabad, Gujarat, India \\ ${ }^{4}$ Department of Radiology, HCG Cancer Centre, Ahmedabad, \\ Gujarat, India
}

\begin{abstract}
Address for correspondence Diva S. Shah, MBBS, MD, HCG Cancer Centre, Sola-Science City Road, Ahmedabad 380060, Gujarat, India (e-mail: drdiva1923@yahoo.co.in).
\end{abstract}

J Gastrointestinal Abdominal Radiol ISGAR 2021;4:154-160.

\begin{abstract}
Autoimmune pancreatitis (AIP) consists of two clinically histologically distinct forms Keywords

- autoimmune pancreatitis

- pancreatic cancer

- pancreatic adenocarcinoma

- mass mimicking autoimmune pancreatitis (type I and II) of chronic pancreatitis that are histologically different. These forms of AIP classically respond to oral steroids. The focal form of AIP resembles pancreatic carcinoma both clinically and radiologically and it is of utmost importance to make an early correct diagnosis between these two diseases in order to identify the optimal therapeutic strategy and to avoid unnecessary laparotomy or pancreatic resection in AIP patients. Here we report focal forms of type I and II AIP with clinical and imaging features closely mimicking pancreatic carcinoma.
\end{abstract}

\section{Introduction}

Autoimmune pancreatitis (AIP) was first described by Sarles et al in 1961 as "primary inflammatory sclerosis" of the pancreas. ${ }^{1}$ However, later it was identified as distinctive subtype of chronic pancreatitis caused by autoimmune inflammatory processes with heavy lymphocyte infiltration and fibrosis that result in organ dysfunction by Yoshita et al in 1995. ${ }^{2}$

It is a rare form of chronic inflammatory pancreatic disease with unclear etiology, but autoimmune mechanisms, genetic susceptibility, and exposure to environmental factors are favored. AIP has two distinct types-type I is considered as pancreatic manifestation of immunoglobulin (IgG4)related multisystem disease, while type II is considered as isolated pancreatic disease independent of IgG4.,4
On imaging, involvement of pancreas can be either diffuse, focal, or multifocal. Focal form of AIP typically presents with clinical and radiological manifestations mimicking pancreatic cancer. ${ }^{3-6}$

Here, we report clinical and imaging features that assist differentiation of type I and type II AIP and also help to differentiate focal form of both types of AIP from pancreatic cancer.

\section{Case Discussion}

\section{Case 1}

A 62-year-old nonalcoholic male presented in gastrosurgery department with chief complaints of backache and mild abdominal pain since 6 to 7 months without significant weight loss and anorexia. Physical examination showed slight deep tenderness in epigastrium; no lump was palpable. published online

March 3, 2021
DOI https://doi.org/

$10.1055 / \mathrm{s}-0041-1722813$

ISSN 2581-9933. (c) 2021. Indian Society of Gastrointestinal and Abdominal Radiology. This is an open access article published by Thieme under the terms of the Creative Commons Attribution-NonDerivative-NonCommercial-License, permitting copying and reproduction so long as the original work is given appropriate credit. Contents may not be used for commercial purposes, or adapted, remixed, transformed or built upon. (https://creativecommons.org/licenses/by-nc-nd/4.0/).

Thieme Medical and Scientific Publishers Pvt. Ltd. A-12, 2nd Floor, Sector 2, Noida-201301 UP, India 
Ultrasonography of abdomen showed a fairly defined hypoechoic mass with necrotic area in head of pancreas and suprapancreatic region with normal diameter of main pancreatic duct (MPD). There was another hypoechoic mass encircling lower part of abdominal aorta and bilateral common iliac arteries.

Triphasic contrast enhanced computed tomography (CECT) of abdomen revealed a hypodense mass lesion in the head of the pancreas and suprapancreatic region with necrotic area in its inferior aspect. It appeared hypodense on arterial phase with inhomogeneous mild enhancement on portal venous phase and appeared iso dense on delayed phase (-Fig. 1A and B). Additionally, a poorly enhancing soft tissue density lesion encircling lower part of abdominal aorta and bilateral common iliac arteries (-Fig. 2) with mild atheromatous changes involving adventitia of abdominal aorta and iliac arteries. A pancreatic malignant lesion with differential diagnosis of lymphoma or retroperitoneal fibrosis along abdominal arteries was given on the basis of contrast enhanced CT scan findings.

Serum levels of cancer antigen (CA) 19.9 and carcinoma embryonic antigen (CEA) were 2.6 (normal range: 0-37 U/mL) and $0.98 \mathrm{ng} / \mathrm{mL}$ (normal value: $<2.5 \mathrm{ng} / \mathrm{mL}$ ), respectively, and mean blood sugar was $114 \mathrm{mg} / \mathrm{dL}$.

Ultrasound-guided biopsy (-Fig. 3) from pancreatic mass lesion showed fibrosis with inflammatory infiltrate with no evidence of malignancy (-Fig. 4). Serum IgG4 level was $180 \mathrm{mg} / \mathrm{dL}$ (normal range: $8-140 \mathrm{mg} / \mathrm{dL}$ ).

\section{Case 2}

A 35-year-old nonalcoholic male presented with chief complaints of off and on pain in abdomen for the last 6 to 7 months with $5 \mathrm{~kg}$ loss of weight, yellowish discoloration of urine, and significant loss of appetite for the last 4 months. He was also recently diagnosed with diabetes mellitus. Physical examination showed slight deep tenderness in epigastrium as well as severe icterus.

Total serum bilirubin was 10.2 (direct: $6.6 \mathrm{mg} / \mathrm{dL}$, indirect: $4.0 \mathrm{mg} / \mathrm{dL}$ ) with serum levels of CA 19.9 and CEA of $103 \mathrm{U} / \mathrm{mL}$ and $1.5 \mathrm{ng} / \mathrm{mL}$, respectively. Mean blood sugar levels were $296 \mathrm{mg} / \mathrm{dL}$ and glycosylated hemoglobin was 10.6 .

Ultrasonography of abdomen showed a fairly-defined hypoechoic mass with prominent MPD. The common bile duct was significantly dilated with its diameter $17 \mathrm{~mm}$ and abrupt cutoff at the level of head of pancreas. Mild prominence of intrahepatic biliary radicals was also observed.

Triphasic CECT scan showed focal isoenhancing ill-defined lesion seen in head of the pancreas. Additionally, the scan showed the bulky distal body and tail of pancreas with peripancreatic thin rim of fluid collection ( - Fig. 5A and B). Since the features of CECT scan were equivocal, magnetic resonance imaging (MRI) was done.

Noncontrast MRI of upper abdomen showed mildly hyperintense mass lesion in head of the pancreas (-Fig. 6A). MRI also showed bulky tail of pancreas with thin hypointense rim seen around distal body and tail of pancreas (-Fig. 6B). The lesion showed restriction on diffusion-weighted imaging with apparent diffusion coefficient (ADC) value ranging from 0.8 to $1.1 \times 10^{-3}$ (-Fig. 7).

Biliary stenting was done after endoscopic retrograde pancreatography and brush cytology was done from ampulla that was negative for malignant cells. Ultrasound guided biopsy from pancreatic head lesion revealed no evidence of malignancy. Serum IgG4 levels were within normal range.

\section{Discussion}

AIP is a distinct form of pancreatitis characterized clinically by frequent presentation with obstructive jaundice with or without a pancreatic mass, histologically by
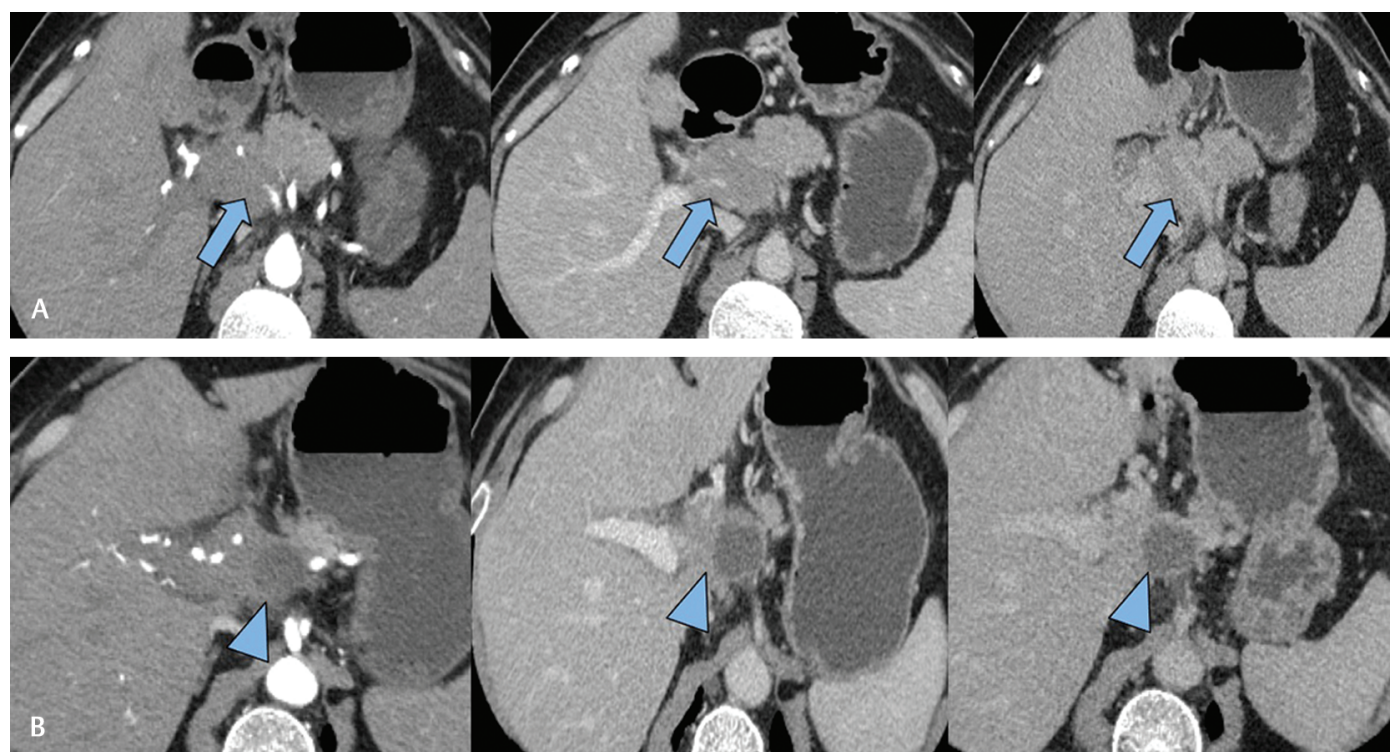

Fig. 1 (A and B) Triphasic axial contrast-enhanced computed tomography scan shows a poorly defined mass lesion involving head of pancreas, appears hypodense on arterial phase, iso to minimally hypodense on portal venous phase, and isodense on delayed phase (arrow) with a necrotic component in its superior aspect (arrowhead); mass is encasing hepatic artery without any luminal narrowing or wall irregularity. 


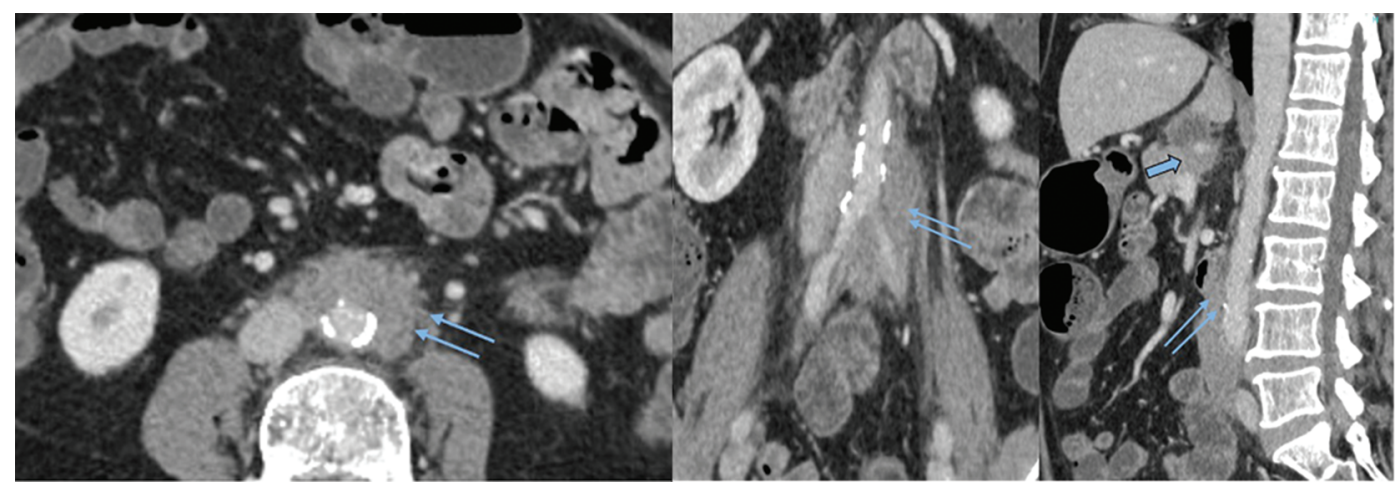

Fig. 2 Axial, coronal, and sagittal reformatted contrast-enhanced computed tomography scan images show soft tissue encircling distal aorta and common iliac arteries (double thin arrow), mass lesion involving head of pancreas (arrow) in sagittal reformatted image.

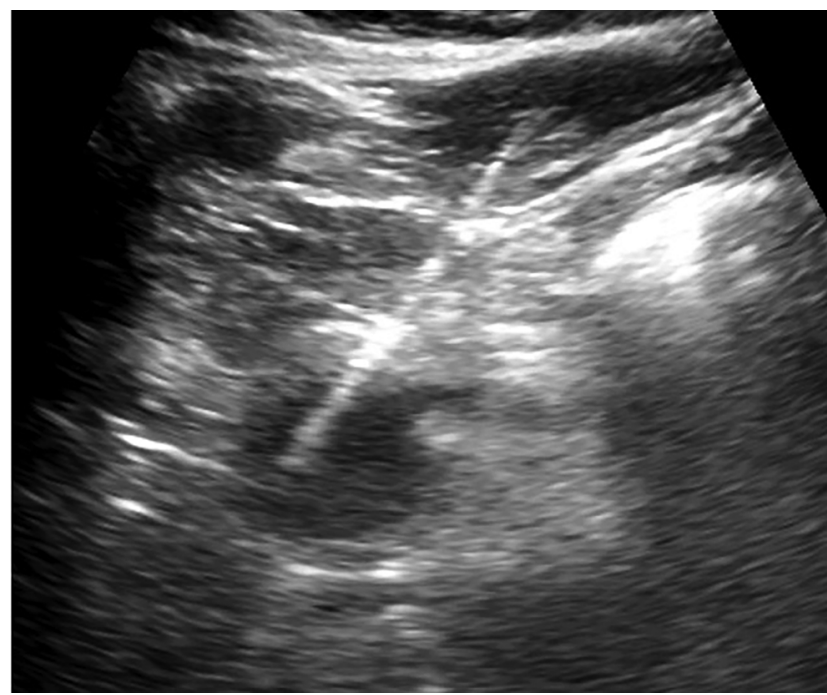

Fig. 3 Ultrasonography with guided biopsy from hypoechoic lesion of head pancreas.

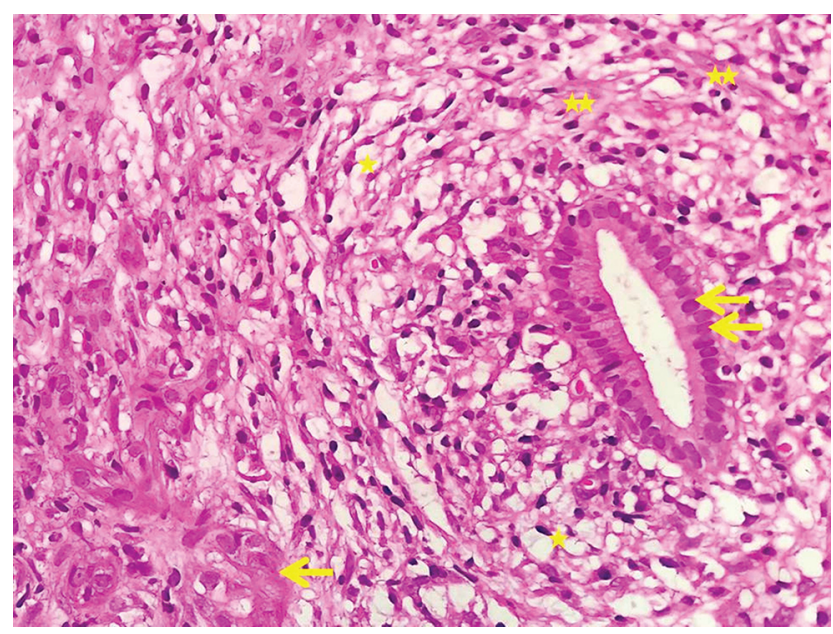

Fig. 4 Pancreatic tissue showing ducts (double arrow) with atrophic changes, few islets (single arrow), and fibrosis (double asterisks) with lymphoplasmacytic inflammatory infiltrate with histiocytes (single asterisk) (hematoxylin and eosin X 400).

lymphoplasmacytic infiltrate with fibrosis, and therapeutically by a dramatic response to steroids. ${ }^{1-5}$
AIP accounts for 5 to $6 \%$ among all chronic pancreatitis. ${ }^{3}$ Men are affected at least twice as often as women, with a reported male-to-female ratio as high as 15:2. ${ }^{4}$ The age at presentation ranges from 14 to 85 years, with the mean age over 60 years. ${ }^{5}$ In our report, both were male patient of 65 and 37 years, respectively.

Focal forms (mass mimicking) AIP accounts for approximately 5 to $11 \%$ of Whipple's resections done with a suspicion of pancreatic adenocarcinoma. ${ }^{6}$ However, with increasing reports of AIP patients in literature, it is possible to diagnose these patients timely preoperatively avoiding unnecessary surgery and associated morbidity. ${ }^{7}$

Typical clinical manifestations of AIP include fluctuating obstructive jaundice, vague abdominal pain, weight loss, occasionally steatorrhea, and diabetes. Diabetes (usually type II) is present in 43 to $68 \%$ of patients. ${ }^{8}$ In present report, patient with type II AIP (case 2 ) had type II diabetes.

\section{Types of AIP}

AIP is divided into type I and II, each one has distinct histopathology, clinical features, and different diagnostic criteria. ${ }^{5-11}$ In 2011, International Association of Pancreatology developed International Consensus Diagnostic Criteria (ICDC) for AIP and proposed two distinct histological of AIP. Type I AIP is also called lymphoplasmacytic sclerosing pancreatitis (LPSP) or AIP without granulocyte epithelial lesions (GEL). LPSP is characterized infiltration of tissue by IgG4 plasma cell with storiform fibrosis and phlebitis. Type II AIP is also defined as idiopathic duct-centric pancreatitis (IDCP) or AIP with GEL. ${ }^{12,13}$

Histologically, type II AIP is characterized by neutrophilic infiltration of epithelium and lumen of pancreatic duct.

Type I AIP (LPSP) seems to be the pancreatic manifestation of an IgG4-related systemic disease and it is characterized by elevated IgG4 serum levels, infiltration of IgG4-positive plasma cells, and extrapancreatic lesions (e.g., sclerosing cholangitis, sclerosing sialadenitis, and retroperitoneal fibrosis). Type I AIP is three times common in males than in females and often seen in sixth decade. ${ }^{10,11,14,15}$

Type II (IDCP) usually has none or very few IgG4-positive plasma cells, no serum IgG4 elevation, and appears to be a pancreas-specific disorder without extrapancreatic 

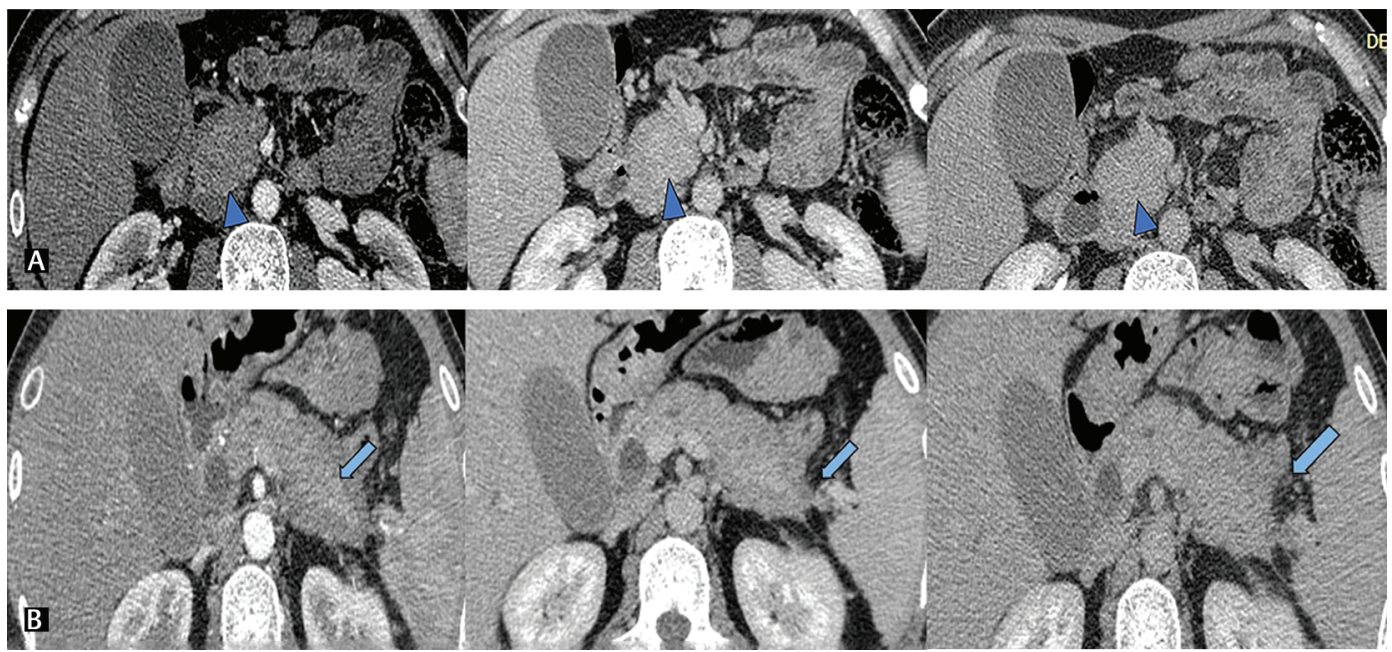

Fig. 5 (A) Triphasic contrast-enhanced computed tomography (CECT) shows Iso enhancing bulky lesion in head of the pancreas (arrowhead). (B) Triphasic CECT scan shows bulky distal body and tail of pancreas with hypodense perifocal rim (marked by arrow).
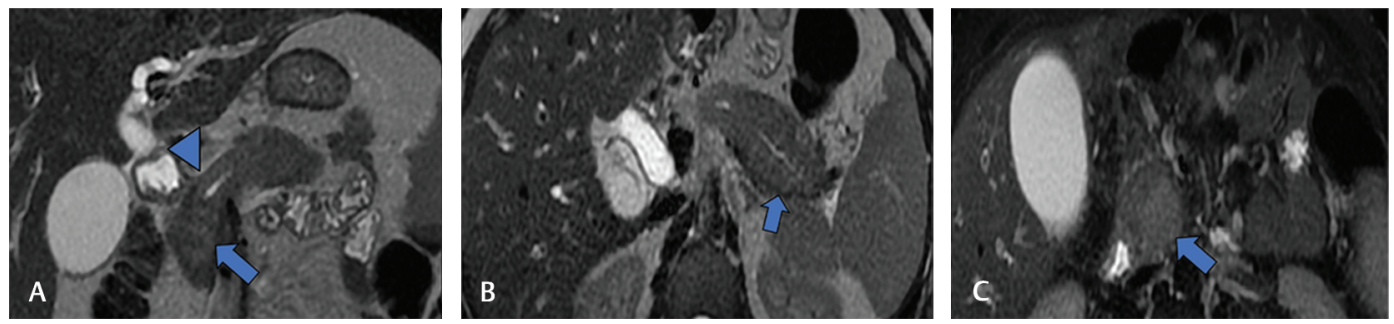

Fig. 6 Axial T2-weighted magnetic resonance imaging (T2W MRI) (A) shows mildly hyperintense mass lesion in head of pancreas with proximal dilatation of biliary duct (large arrow and arrowhead, respectively). Axial T2W MRI (B) shows bulky tail of pancreas with hypointense "capsule rim sign" (small arrow), and T2WFS image (C) shows focal mildly hyperintense mass lesion in head of pancreas on T2WFS images (arrow).

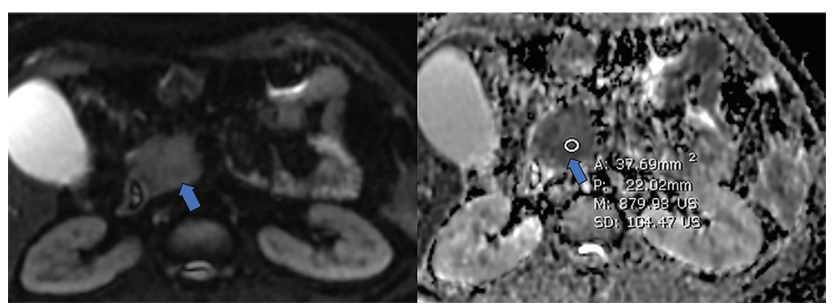

Fig. 7 Axial diffusion-weighted imaging and apparent diffusion coefficient (ADC) map shows diffusion restriction (small arrow) in lesion of head of the head of pancreas with ADC value $0.87 \times 10^{-3} \mathrm{~mm}^{2} / \mathrm{s}$.

involvement. Type II AIP is commonly seen in younger patients between 20 and 50 years with mean age 45 years without any gender preponderance. It is often associated with inflammatory bowel disease and with diabetes. ${ }^{10,11,14,15}$

IgG4-related sclerosing disease can involve various organs including biliary tree, kidneys, gallbladder, retroperitoneum, mesentery, blood vessels, lungs, orbits, lymph nodes, salivary glands, thyroid gland, and prostate gland. ${ }^{12,14}$

Retroperitoneal fibrosis is present in about 10 to $20 \%$ of patients with type I AIP and it is characterized by a thick soft-tissue mass that is confined to the retroperitoneum and pelvic brim and typically covers the abdominal aorta. ${ }^{14}$
In present report, the first case of elderly gentleman had focal involvement of pancreatic head, retroperitoneal fibrosis, and elevated serum IgG4 levels suggesting type I AIP with both pancreatic and retroperitoneal fibrosis as IgG4-related extrapancreatic manifestation.

However, second case of a young male showed an isolated involvement of pancreas with normal IgG4 levels. No extrapancreatic manifestations were observed that signifies type II variety of AIP.

Elevated serum IgG4 levels are characteristic in AIP but serum IgG4 serology is not the sole criteria to differentiate AIP from pancreatic carcinoma. More than $20 \%$ of all AIP have normal Ig4 levels, particularly type II AIP and nearly 7 to $10 \%$ of the patients of pancreatic carcinoma have elevated IgG4 levels. ${ }^{13}$ Types of AIP have been summarized in - Table 1 .

\section{Imaging Characteristics}

Three distinct patterns of AIP are recognized in literature: diffuse, focal, and multifocal. ${ }^{11,12,14,16}$ Diffuse form is the commonest pattern, characterized by "sausage-like" pancreatic enlargement with sharp margins and loss of the lobular contour, while focal form is less common that accounts for 33 to $41 \%$ of all cases of AIP. ${ }^{16}$ Multifocal involvement is least common form of AIP, it can involve head, body, or tail of pancreas with presence of multiple areas of pancreatic duct narrowing. ${ }^{11,16}$ Pancreatic head 
Table 1 Difference between types of AIP

\begin{tabular}{|l|l|l|}
\hline Types of AIP & Type 1 & Type 2 \\
\hline $\begin{array}{l}\text { Histological } \\
\text { name }\end{array}$ & $\begin{array}{l}\text { Lymphoplasmacytic } \\
\text { sclerosing pancreatitis }\end{array}$ & IDCP \\
\hline Age/gender & Adult, middle aged/M>F & Young/M=F \\
\hline Clinical features & $\begin{array}{l}\text { Obstructive jaundice, } \\
\text { rarely abdominal pain }\end{array}$ & $\begin{array}{l}\text { Obstructive } \\
\text { jaundice, often } \\
\text { abdominal pain, } \\
\text { weight loss }\end{array}$ \\
\hline $\begin{array}{l}\text { Extrapancreatic } \\
\text { organ } \\
\text { involvement }\end{array}$ & $\begin{array}{l}\text { Common-biliary tree } \\
\text { (sclerosing cholangitis) } \\
\text { retroperitoneal fibrosis, } \\
\text { sclerosing mesenteritis, } \\
\text { sialadenitis, arteritis, } \\
\text { and rarely autoimmune } \\
\text { prostatitis }\end{array}$ & Unusual \\
\hline $\begin{array}{l}\text { Steroid } \\
\text { response }\end{array}$ & $\begin{array}{l}\text { Response-partial } \\
\text { response and rate of } \\
\text { recurrence are more }\end{array}$ & $\begin{array}{l}\text { Response- } \\
\text { complete } \\
\text { response }\end{array}$ \\
\hline Relapse & Common & Rare \\
\hline Serology Ig4 & High & Normal \\
\hline Associations & Not known & $\begin{array}{l}\text { Ulcerative colitis, } \\
\text { Type II diabetes }\end{array}$ \\
\hline
\end{tabular}

Abbreviations: AIP, autoimmune pancreatitis; IDCP, idiopathic duct-centric pancreatitis.

is the most common site for the focal involvement in AIP and appears as hypoechoic lesion on ultrasonography. The differentiation is extremely difficult as this appearance is not specific and often seen in pancreatic carcinoma and in other forms of chronic mass forming inflammatory pancreatitis.

Cross-sectional imaging of pancreas is the very important to diagnose AIP. The CT imaging features in AIP include (a) diffuse swelling of the pancreas, which is referred to as a "sausage-like" or "bulky" appearance, (b) a capsule-like smooth rim of low density surrounding the pancreas that appears to be characteristic of AIP and related to inflammatory and fibrotic changes in peripancreatic fat, (c) absence of pancreatic calcification and intraductal stones, and (d) delayed enhancement of affected pancreatic lesion. ${ }^{11,12,14,16}$

Muhi et $a{ }^{16}$ defined seven criteria to differentiate mass-forming AIP from pancreatic carcinoma with high accuracy based on CECT/MR in various combinations. This includes (1) early homogeneous good enhancement, (2) delayed homogeneous good enhancement, (3) hypoattenuating capsule-like rim, (4) MPD upstream diameter $<4 \mathrm{~mm}$, (5) the absence of distal pancreatic atrophy, (6) "duct penetrating sign"-a result of inflammatory nature of AIP causing duct narrowing as against complete obstruction of duct in pancreatic carcinoma, and (7) ADC value $\leq 0.87 \times 10^{-3 \mathrm{~mm} 2} / \mathrm{s}$. In present report, in both the cases 5 out of 7 signs were seen including delayed homogenous enhancement, capsule rim sign, MPD upstream diameter $<4 \mathrm{~mm}$, absence of distal pancreatic atrophy, and lower ADC value.

The CT attenuation of the pancreatic lesion AIP is similar to or greater than that of the liver in both the pancreatic and the hepatic phases. ${ }^{17,18}$ Wakabayashi et al evaluated the CT enhancement pattern of a focal form of AIP in nine patients. In six out of nine patients, the lesions were hypoattenuating in the early phase but were homogeneously isoattenuating in the delayed phase and also suggested that pancreatic carcinoma had decreased enhancement in the pancreatic phase and minimal change in enhancement in the hepatic phase. This finding may help differentiate the focal form of AIP from pancreatic carcinoma. ${ }^{18}$

In present case report, one patient showed hypoattenuation in pancreatic phase, while another patient showed isoattenuation in pancreatic phase and both showed isoenhancement in delayed phase.

On MRI, focal forms of AIP show T1 hypointensity and mild T2 hyperintensity. MRI shows "capsule rim" sign more accurately. AIP tends to have greater diffusion restriction with lower ADC values as compared to pancreatic adenocarcinoma and normal pancreatic parenchyma. The average ADC value suggested in AIP is $\leq 0.87 \times 10^{-3}$ by Muhi et al.16 Similarly, Kamisawa et al also found lower ADC values in AIP patients $\left(1.012 \pm 0.112 \times 10^{-3} \mathrm{~mm}^{2} / \mathrm{s}\right)$ than in pancreatic cancer patients $\left(1.2496 \pm 0.113 \times 10^{-3} \mathrm{~mm}^{2} / \mathrm{s}\right) .{ }^{19}$

In present report, second patient (type II AIP) showed "capsule rim sign," focal mild T2-weighted hyperintensity, and $A D C$ value in focal lesion is significantly lower than $0.87 \mathrm{x}$ $10^{-3} \mathrm{~mm}^{2} / \mathrm{s}$.

Dynamic MRI with secretin administration has potential role in differentiation from benign inflammatory stricture of MPD in AIP from obstructive stricture of pancreatic adenocarcinoma. Patency of inflammatory duct can be demonstrated after secretin stimulation that is known as "duct penetrating sign." 20 Difference between AIP and pancreatic carcinoma has been shown in schematic diagram (-Fig. 8).

Conventional endoscopic ultrasonography (EUS) plays an important in identifying small pancreatic lesion. However, EUSguided fine-needle aspiration has limited role in diagnosis of nonmalignant pancreatic parenchymal conditions; therefore, image-guided percutaneous biopsy preferred when lesion is well demonstrated on imaging studies and in case of smaller lesion or lesion EUS-guided core biopsy can be alternative. ${ }^{21}$
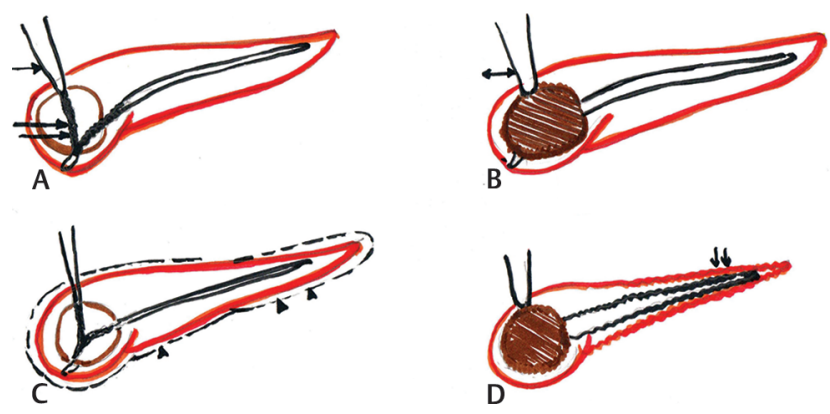

Fig. 8 Schematic diagram shows imaging appearance of mass mimicking autoimmune pancreatitis versus pancreatic malignancy. (A) "Penetrating duct sign" (irregular and narrowed duct seen through lesion (double arrow) with mild upstream dilatation of common bile duct (single arrow) in mass mimicking AIP. (B) "Double duct sign" with cutoff of ducts in malignant lesion of head of pancreas. (C). Bulky rest of the pancreas with "capsule rim sign" (arrowhead). (D) Carcinoma of head of pancreas with atrophic distal pancreatic parenchyma and dilated irregular pancreatic duct 

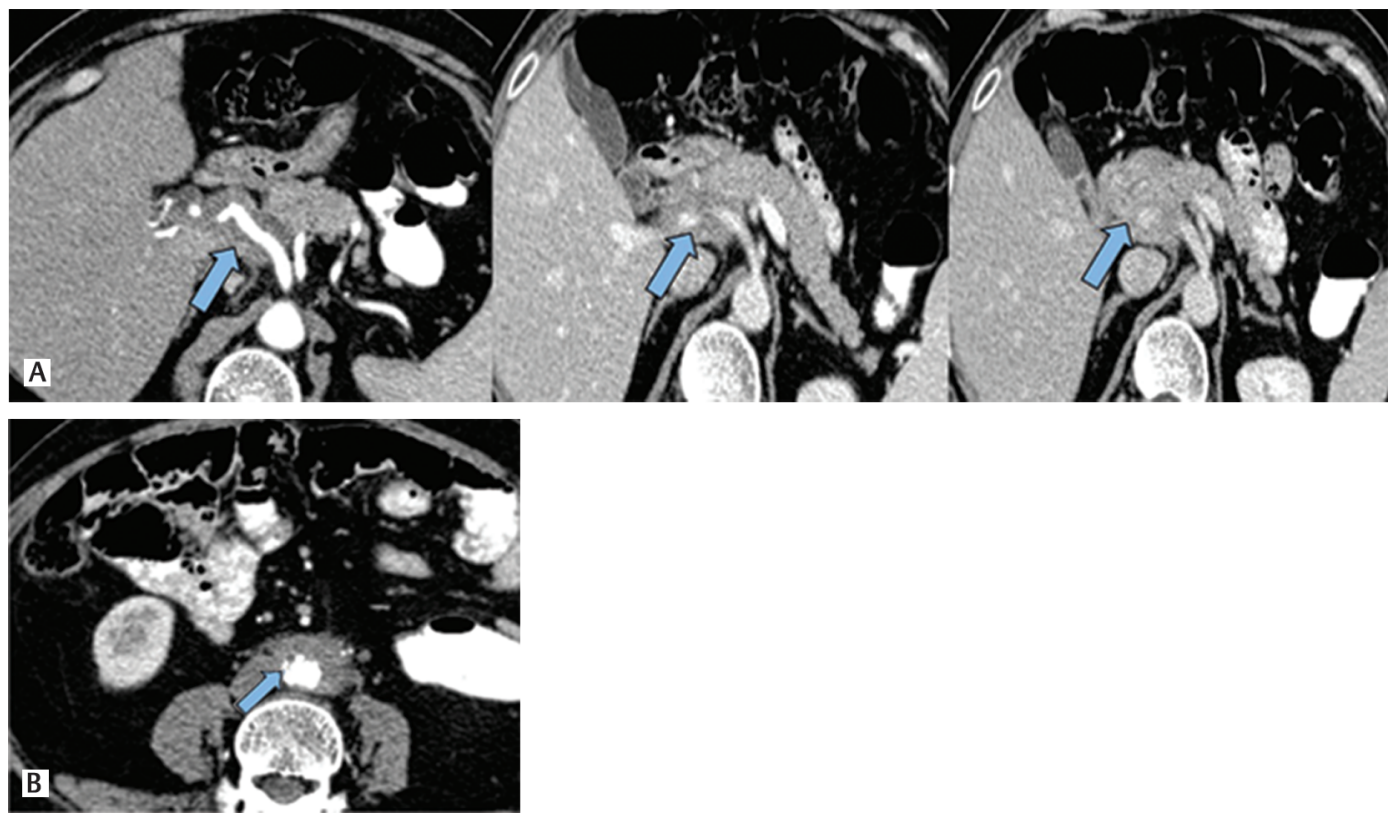

Fig. 9 (A and B) Follow-up triphasic contrast-enhanced computed tomography scan after steroidal therapy shows residual lesion in head of the pancreas (large arrow) and mild regression in bulk of retroperitoneal fibrosis (thin arrow).

\section{Follow-Up and Treatment}

Both subtypes of AIP show dramatic clinical response after oral corticosteroid treatment that is evident by returning of normal morphological and pancreatic functions as well as resolution of pancreatic duct narrowing or irregularity in 4 to 6 weeks. Follow-up with multidetector computed tomography (MDCT) or MRI imaging helps in response evaluation that evaluates the regression in size of pancreatic lesion or regression in bulk of pancreas. However, type I AIP with focal involvement has higher risk of recurrence or poor treatment response. ${ }^{19,20}$ Follow-up MDCT of one our patient (type I) showed mild response to corticosteroid therapy (-Fig. 9A and B). Recently, in corticosteroid resistant or relapse cases of AIP, few immunomodulators such as azathioprine, cyclosporine, and rituximab have been suggested. 22,23

\section{Conclusion}

AIP is rare but not an exceptional entity. Precise radiological distinction by various imaging features, pattern of contrast enhancement on CT scan, and timely diagnosis of AIP is very crucial to avoid unnecessarily pancreatic resection and major abdominal surgeries; thus, associated comorbidities and speedy recovery are avoided. Clinical and imaging features also helps to differentiate type I and II variety of AIP. It is essential for radiologist to know precise imaging signs of focal forms of both types of AIP to differentiate from pancreatic carcinoma.

\section{Conflict of Interest}

None declared.

\section{References}

1 Sarles H, Sarles JC, Muratore R, Guien C. Chronic inflammatory sclerosis of the pancreas-an autonomous pancreatic disease? Am J Dig Dis 1961;6(7):688-698

2 Yoshida K, Toki F, Takeuchi T, Watanabe S, Shiratori K, Hayashi N. Chronic pancreatitis caused by an autoimmune abnormality. Proposal of the concept of autoimmune pancreatitis. Dig Dis Sci 1995;40(7):1561-1568

3 Khandelwal A, Inoue D, Takahashi N. Autoimmune pancreatitis: an update. Abdom Radiol (NY) 2020;45(5):1359-1370 [Internet]

$4 \mathrm{Kim} \mathrm{KP}$, Kim MH, Song MH, Lee SS, Seo DW, Lee SK. Autoimmune chronic pancreatitis. AmJGastroenterol2004;99(8):1605-1616

5 Chari ST. Diagnosis of autoimmune pancreatitis using its five cardinal features: introducing the Mayo Clinic's HISORt criteria. J Gastroenterol 2007;42(Suppl 18) :39-41

6 Yadav D, Notahara K, Smyrk TC, et al. Idiopathic tumefactive chronic pancreatitis: clinical profile, histology, and natural history after resection. Clin Gastroenterol Hepatol 2003;1(2):129-135

7 Psarras K, Baltatzis ME, Pavlidis ET, Lalountas MA, Pavlidis TE, Sakantamis AK. Autoimmune pancreatitis versus pancreatic cancer: a comprehensive review with emphasis on differential diagnosis. Hepatobiliary Pancreat Dis Int 2011;10(5):465-473

8 Okazaki K, Chiba T. Autoimmune related pancreatitis. Gut 2002;51(1):1-4

9 Lo RSC, Singh RK, Austin AS, Freeman JG. Autoimmune pancreatitis presenting as a pancreatic mass mimicking malignancy. Singapore Med J 2011;52(4):e79-e81

10 Chari ST, Kloeppel G, Zhang L, Notohara K, Lerch MM, Shimosegawa T. Histopathologic and clinical subtypes of autoimmune pancreatitis: the Honolulu consensus document. Pancreatology 2010;10(6):664-672 
11 Crosara S, D'Onofrio M, De Robertis R, et al. Autoimmune pancreatitis: multimodality non-invasive imaging diagnosis. World J Gastroenterol 2014;20(45):16881-16890

12 Gill M, Brar K, Godara R, et al. Autoimmune pancreatitis masquerading as carcinoma head of pancreas: a case report and review of literature. Ann Med Surg (Lond) 2019;45:82-85

13 Shimosegawa T, ChariST, Frulloni L, etal; International Association of Pancreatology. International consensus diagnostic criteria for autoimmune pancreatitis: guidelines of the International Association of Pancreatology. Pancreas 2011;40(3):352-358

14 Van Moerkercke W, Verhamme M, Doubel P, Meeus G, Oyen R, Van Steenbergen W. Autoimmune pancreatitis and extrapancreatic manifestations of IgG4-related sclerosing disease. Acta Gastroenterol Belg 2010;73(2):239-246

15 Cao Z, Tian R, Zhang T, Zhao Y. Localized autoimmune pancreatitis: report of a case clinically mimicking pancreatic cancer and a literature review. Medicine (Baltimore) 2015;94(42):e1656

16 Muhi A, Ichikawa T, Motosugi U, et al. Mass-forming autoimmune pancreatitis and pancreatic carcinoma: differential diagnosis on the basis of computed tomography and magnetic resonance cholangiopancreatography, and diffusion-weighted imaging findings. J Magn Reson Imaging 2012;35(4):827-836
17 Vijayakumar A, Vijayakumar A. Imaging of focal autoimmune pancreatitis and differentiating it from pancreatic cancer. ISRN Radiol 2013;2013:569489

18 Wakabayashi T, Kawaura Y, Satomura Y, et al. Clinical and imaging features of autoimmune pancreatitis with focal pancreatic swelling or mass formation: comparison with so-called tumor-forming pancreatitis and pancreatic carcinoma. Am J Gastroenterol 2003;98(12):2679-2687

19 Kamisawa T, Takuma K, Anjiki H, et al. Differentiation of autoimmunepancreatitisfrompancreaticcancerbydiffusion-weighted MRI. Am J Gastroenterol 2010;105(8):1870-1875 [Internet]

20 Negrelli R, Boninsegna E, Avesani G, etal.Type 1 and type 2 autoimmune pancreatitis: distinctive clinical and pathological features, but are there any differences at magnetic resonance? Experience from a referral center. Pancreas 2018;47(9):1115-1122

21 Chhoda A, Rustagi T. EUS-guided needle biopsy for autoimmune pancreatitis. Clin J Gastroenterol 2020;13(5):669-677 [Internet]

22 Gardner TB, Chari ST. Autoimmune pancreatitis. Gastroenterol Clin North Am 2008;37(2):439-460, vii

23 Hart PA, Kamisawa T, Brugge WR, et al. Long-term outcomes of autoimmune pancreatitis: a multicentre, international analysis. Gut 2013;62(12):1771-1776 